\title{
Agir en situation d'incertitude : le cas des éleveurs de bovins allaitants
}

Beef cattle farmer's strategies in the face of uncertainty

\section{Bruno Lemery, Stéphane Ingrand, Benoît Dedieu et Béatrice Dégrange}

\section{(2) OpenEdition}

Édition électronique

URL : http://journals.openedition.org/economierurale/2718

DOI : $10.4000 /$ economierurale. 2718

ISSN : 2105-2581

Éditeur

Société Française d'Économie Rurale (SFER)

Édition imprimée

Date de publication : 2 août 2005

Pagination : 57-69

ISSN : 0013-0559

Référence électronique

Bruno Lemery, Stéphane Ingrand, Benoît Dedieu et Béatrice Dégrange, « Agir en situation

d'incertitude : le cas des éleveurs de bovins allaitants », Économie rurale [En ligne], 288 | Juillet-août 2005, mis en ligne le 05 juillet 2009, consulté le 21 avril 2019. URL : http://journals.openedition.org/ economierurale/2718; DOI : 10.4000/economierurale.2718

(c) Tous droits réservés 


\section{$A_{\text {gires sistation frinectitude: }}$ le cas des éleveurs de bovins allaitants}

Bruno LEMERY • Enesad, Département économie et sociologie, Centre Inra de Dijon, Ur Listo Stéphane INGRAND, Benoît DEDIEU • Centre Inra de Theix, Umr Metafort Béatrice DEGRANGE • Enesad, Département formation et communication

La globalisation de l'économie, d'un côté, la diversification des demandes adressées au secteur agricole, de l'autre, se traduisent par une remise en cause du modèle de développement autour duquel ce secteur s'était progressivement organisé. Les agriculteurs sont ainsi invités à un effort d'adaptation. Cette notion ne va cependant pas de soi. Elle induit l'idée d'une réalité déjà constituée, alors que ce à quoi il s'agit de s'adapter fait question. Elle tend à occulter le fait que le devenir de l'agriculture dépend de certaines prises de position relatives à ce que devrait être cette activité même. S'interroger sur l'adaptation des agriculteurs aux évolutions de leur environnement suppose alors de prêter attention, d'une part, à la façon dont ceux-ci appréhendent ces évolutions et conçoivent, à partir de là, ce qu'il convient éventuellement de changer dans la conduite de leurs exploitations et, d'autre part, aux problèmes pratiques que pose l'engagement de tels changements dans une situation d'incertitude. C'est suivant une telle perspective que nous avons effectué ${ }^{1}$ une étude des réactions des éleveurs de bovins allaitants aux perturbations que connaît actuellement leur monde de pro-

1. Dans le cadre d'un programme mis en place à l'initiative du Centre INRA de Dijon et de la Délégation à l'animation au développement et à la prospective (DADP) de cet Institut, programme de recherches «Pour et Sur le Développement Régio$n a l »$ (PSDR Bourgogne). duction, étude pluridisciplinaire menée par une équipe associant des sociologues, des économistes et des zootechniciens ${ }^{2}$.

Dans cet article, après avoir explicité les modalités de construction de cette recherche, nous exposons ses résultats principaux $^{3}$ et nous proposons, pour conclure, quelques réflexions sur les enseignements que l'on peut en tirer quant à la manière d'envisager un accompagnement susceptible de contribuer à accroître la capacité d'action des éleveurs.

\section{Problématique et dispositif de recherche}

\section{Une recherche centrée}

sur les transformations " en actes " de l'élevage

La modernisation de l'agriculture française initiée dans l'après-guerre a abouti à la constitution d'un secteur d'activité spécialisé dans la production de matières premières alimentaires. Depuis les années 1980, se secteur rencontre une série de blocages dans ses mécanismes internes de développement (crises de surproduction, contestation de la légitimité des financements publics

2. Outre les auteurs de ce texte, sociologues et zootechniciens, ont participé à cette étude Jacques Brossier, économiste, directeur de recherche à l'INRA, et Hélène Bardey, doctorante en économie. 3. Pour une présentation plus détaillée, voir Lémery et al., 2004. 
dont il bénéficie...). Il est, en outre, confronté à la montée de nouvelles demandes sociales, en matière de protection de l'environnement, de sécurité sanitaire des produits ou de préservation des aménités rurales. Si les exigences qu'il se doit intégrer en conséquence - compétitivité sur des marchés de plus en plus ouverts, qualité et traçabilité, durabilité, multifonctionnalité... - commencent à se préciser, on a affaire à une situation assez confuse. Les mots d'ordre par lesquels se traduisent ces exigences ne font qu'indiquer ce vers quoi il faudrait aller et leur compatibilité est, par ailleurs, rien moins qu'évidente.

Dans ces conditions, autant que d'adaptation de l'agriculture à un nouveau contexte, c'est d'invention d'une nouvelle agriculture qu'il faut parler (Lémery, 2003). Ce que l'agriculture est susceptible de devenir dépend d'une certaine définition de ce qu'elle doit être, et cette définition n'est pas déjà là. Elle fait objet de débats dont l'issue est incertaine. Son élaboration suppose tout un processus d'expérimentation technique, économique et social. Pour appréhender le devenir de la production agricole, il ne suffit donc pas de s'attacher à caractériser les différentes forces qui contribuent à en modifier le cadre d'exercice. Il faut aussi examiner comment le sens que les acteurs concernés attribuent à ce qui leur arrive et les actions, parfois antagonistes, dans lesquelles ils s'engagent à partir de là participent à la reconfiguration de cette production. Étudier les évolutions de l'agriculture implique alors de s'intéresser, non pas seulement à ce qui change dans son environnement, mais aussi à l'activité propre de ceux qui y exercent pour faire face aux différents évènements qui viennent aujourd'hui perturber cet exercice et aux prescriptions qui leur sont adressées sur la manière de répondre à ces évènements.

C'est sur ces bases que nous avons conçu une recherche effectuée sur le monde de l'élevage bovin allaitant charolais, terrain choisi pour des raisons d'opportunité, compte tenu de la composition de notre équipe, mais aussi au regard de son caractère bien approprié au type d'étude que nous entendions mener. Ce monde était en effet soumis à des perturbations particulièrement vives au moment où nous avons engagé notre travail, effectué, entre 2001 et 2004, sur une période marquée par la crise de l'Encéphalopathie spongiforme bovine (ESB). Il est également animé par de vifs débats quant aux orientations à lui donner, la crise qu'il a ainsi traversé donnant lieu à des prises de position assez tranchées entre tenants d'un effort de modernisation supplémentaire pour dépasser le caractère « artisanal » de la filière et tenants d'un retour à la «tradition $»^{4}$.

Pour rendre compte de ce que changer veut dire - signifie et implique concrètement - pour les éleveurs, nous nous sommes attachés, plus précisément, à la manière dont ceux-ci gèrent la double contrainte que génère toute situation d'incertitude : répondre à des pressions d'adaptation de plus en plus fortes tout en maintenant des cadres de référence suffisamment stables pour savoir quoi faire et comment. Par certains côtés, notre étude s'inscrit ainsi dans un courant de recherches menées sur les conditions et les modalités de la flexibilité des entreprises. Mais elle s'en distingue aussi par le souci de prendre de la distance avec le genre « d'impératif adaptatif » qui soustend le plus souvent ces travaux ${ }^{5}$.

Telle que théorisée dans le domaine des sciences de gestion (Cohendet et Llerena, 1999 ; Tarondeau, 1999), la notion de flexibilité renvoie assez exclusivement, en effet, aux capacités à faire évoluer la structure et les projets d'une entreprise pour répondre aux évolutions de l'environnement (flexibilité stratégique) et aux capacités à ajuster les compétences ou modifier les méthodes

4. Sur ce point, voir Dégrange, 2001.

5. Impératif dont le caractère idéologique a donné lieu à de nombreuses critiques, dans le champ de la sociologie notamment (De Gaulejac, 2005 ; Barbier et Nadel, 2000 ; Sennett, 2000 ; Boltanski et Chiapello, 1999). 
pour répondre à des variations non anticipées dans cet environnement (flexibilité opérationnelle). Elle met donc l'accent sur l'intégration de contraintes et la saisie d'opportunités externes et elle laisse un peu de côté la question du fond de normes en fonction desquelles ces contraintes et ces opportunités sont évaluées. Moins qu'à la flexibilité des exploitations d'élevage en elle-même, c'est alors au traitement des tensions (Périlleux, 2001) existant entre la nécessité, pour les éleveurs, de modifier leurs systèmes d'exploitation pour faire face aux évolutions actuelles de leur secteur et celle de conserver aussi à ce système une certaine identité qui leur permette de « s'y retrouver », que nous nous sommes intéressés.

\section{Une recherche reposant sur le croisement de points de vue disciplinaires multiples}

Suivant une telle problématique, nous avons organisé nos investigations autour de trois objectifs :

- qualifier la diversité des rapports que les éleveurs entretiennent avec les exigences d'adaptation qui leur sont aujourd'hui adressées, compte tenu de la façon différenciée dont ils y sont exposés et dont ils les interprètent ;

- analyser la façon dont ces différents rapports se traduisent dans la conduite effective de l'activité d'élevage, en étudiant la manière dont les éleveurs procèdent à des réagencements ou à des changements de pratiques, moyennant quels raisonnements, correspondant à quelles logiques ;

- examiner ce que ces logiques et leur distribution au regard des caractéristiques des exploitations et des exploitants révèlent quant à la nature des problèmes rencontrés par les éleveurs dans ces changements ou ces réagencements et quant aux « ressorts » qu'ils mobilisent dans le traitement de ces problèmes.

Pour atteindre ces objectifs, notre recherche impliquait de satisfaire à deux conditions :

- couvrir la diversité des situations des éleveurs au regard de l'adaptation attendue de leur part à un nouveau contexte de production ; - accéder à une compréhension fine des pratiques qu'ils mettent en œuvre à cette fin, pratiques qu'il nous fallait donc appréhender dans toutes leurs dimensions.

Notre dispositif d'étude a ainsi reposé sur le croisement d'approches disciplinaires multiples, moyennant une série d'enquêtes spécifiques successivement menées auprès d'une quinzaine d'exploitations du département de Saône-et-Loire' .

Le choix de ces exploitations a été effectué avec les partenaires professionnels mobilisés pour la réalisation de notre projet $^{7}$, en fonction de deux critères princi$\operatorname{paux}^{8}$ : naisseur $v s$ naisseur-engraisseur, pour prendre en compte la diversité des systèmes de production présents sur notre zone d'étude ; appartenance à un Groupement $v s$ appartenance à 1 ' $\mathrm{AE} 71$, pour prendre en compte la diversité des positions des éleveurs au regard d'un débat entre « commerce organisé » et « commerce libre » assez marqué dans le monde de l'élevage bovin allaitant. Les enquêtes réalisées sur cet échantillon (tableau 1) ont consisté en cinq entretiens successifs, d'une durée de 2 à 3 heures chacun.

- Le premier était destiné à rassembler les informations de base nécessaires pour une caractérisation globale des exploitations. Il nous a aussi servi à préciser, à partir d'un relevé et d'une première analyse des changements opérés par les exploitants depuis leur installation, les points à creuser dans nos enquêtes ultérieures.

6. En 1997, $45 \%$ des exploitations spécialisées en élevage que comptait la Région Bourgogne étant situées dans ce département.

7. Les Groupements de producteurs intervenant sur ce département, l'Association des éleveurs (AE) de Saône-et-Loire et la Chambre d'Agriculture. 8. Au-delà de ces critères, nous avons cherché également à disposer d'une variété de cas de figure en termes de trajectoires d'exploitation («stabilité » $v s$ « changement ») et de dimension du collectif de travail. 
Tableau 1. Principales caractéristiques des élevages enquêtés

\begin{tabular}{l|c|c|c|c|c|c|c|c}
\hline AE & $\begin{array}{c}\text { Année } \\
\text { instal. }\end{array}$ & $\begin{array}{c}\text { SAU } \\
\text { (ha) }\end{array}$ & $\begin{array}{c}\text { dont } \\
\text { culture } \\
\text { (ha) }\end{array}$ & $\begin{array}{c}\text { Vêlages } \\
\text { (nb/an) }\end{array}$ & $\begin{array}{c}\text { Chargement } \\
\text { (UGB/ha SFP) }\end{array}$ & $\begin{array}{c}\text { Orientation } \\
\text { Naisseur (N) } \\
\text { Engraisseur (E) }\end{array}$ & $\begin{array}{c}\text { Mode de } \\
\text { commercialisation } \\
\text { des animaux }\end{array}$ & PCB \\
\hline 1 & 1986 & 101 & 15 & 66 & 1,2 & $\mathrm{~N}$ & GP & 2 \\
\hline 2 & 1990 & 180 & 12 & 180 & 1,7 & $\mathrm{~N}$ & GP & 3 \\
\hline 3 & 1985 & 113 & 25 & 49 & 1,02 & $\mathrm{~N}$ & $\mathrm{GP}$ & 2 \\
\hline 4 & 1984 & 210 & 21 & 145 & 1,4 & $\mathrm{~N}$ & $\mathrm{AE71}$ & 3 \\
\hline 5 & 1996 & 101 & 3 & 62 & 1,2 & $\mathrm{~N}$ & $\mathrm{GP}$ & 2 \\
\hline 6 & 1976 & 125 & 0 & 74 & 1,2 & $\mathrm{NE}$ & $\mathrm{GP}$ & 2 \\
\hline 7 & 1988 & 75 & 11 & 60 & 1,9 & $\mathrm{NE}$ & $\mathrm{GP}$ & 1 \\
\hline 8 & 1978 & 73 & 11 & 53 & 2,2 & $\mathrm{NE}$ & $\mathrm{GP}$ & 2 \\
\hline $9^{*}$ & 1984 & 170 & 25 & 145 & 1,92 & $\mathrm{NE}$ & $\mathrm{GP}$ & - \\
\hline 10 & 1980 & 82 & 9 & 67 & 1,75 & $\mathrm{NE}$ & $\mathrm{AE71}$ & 2 \\
\hline 11 & 1980 & 206 & 111 & 87 & 1,4 & $\mathrm{~N}$ & $\mathrm{AE71}$ & 1 \\
\hline 12 & 1995 & 125 & 25 & 84 & 1,7 & $\mathrm{NE}$ & $\mathrm{AE71}$ & 2 \\
\hline 13 & 1997 & 80 & 0 & 59 & 1,4 & $\mathrm{~N}$ & $\mathrm{AE71}$ & 1 \\
\hline 14 & 1997 & 120 & 4 & 40 & 1,3 & $\mathrm{~N}$ & $\mathrm{AE71}$ & 1 \\
\hline 15 & 1995 & 188 & 38 & 110 & 1,3 & $\mathrm{~N}$ & $\mathrm{GP}$ & 2 \\
\hline
\end{tabular}

* L'éleveur 9 s'est retiré du dispositif après la première enquête ;

SAU : Surface agricole utile ; UGB : Unité gros bétail ; SFP : Surface fourragère permanente ; GP : Groupement de producteurs ; AE 71 : Association des éleveurs de Saône-et-Loire ; PCB : Personnes dans la cellule de base (personnes tirant un revenu du travail dans l'exploitation, hors salariés)

- Le deuxième devait nous permettre de recueillir les informations nécessaires pour une analyse de l'organisation technique de l'activité d'élevage, c'est à dire de l'ensemble des décisions qui permettent de renouveler le troupeau (nombre et composition) et de le faire produire (volume de production, types de produits et répartition dans le temps) $)^{9}$. Faisant l'hypothèse que toute préconisation ou toute intention de changement met en cause l'organisation de la conduite du troupeau (alimentation, reproduction, tris/allotement), nous avons relevé les changements que celle-ci avait pu connaître au cours de l'histoire de l'exploitation afin de caractériser les raisons et les modalités de ces changements et d'en évaluer les conséquences sur la cohérence et les

9. Notre approche s'inscrivait là dans le prolongement des recherches agronomiques menées sur les pratiques et les systèmes de pratiques des agriculteurs (Cristofini et al., 1978 ; Landais et Deffontaines, 1988 ; Sébillotte et Soler, 1990). marges de manœuvre du système technique. - Le troisième visait à obtenir les informations nécessaires pour une analyse des pratiques économiques et commerciales des exploitants enquêtés. Il s'agissait de mettre en évidence les variables conditionnant la capacité de réponse de l'éleveur aux évolutions du marché - et à la demande d'animaux de qualité, en particulier. Il s'agissait aussi de se faire une idée des raisonnements adoptés par les éleveurs au regard de ces évolutions. Le but de cette double analyse était de confronter le potentiel économique des éleveurs à leurs pratiques de gestion effectives et de caractériser les écarts pouvant exister entre ce potentiel et ce qu'ils en font.

- Le quatrième a été mené de manière à rassembler les informations nécessaires pour une analyse des pratiques d'organisation $d u$ travail en partant du principe que les transformations de l'élevage impliquent des changements dans l'agencement des diverses tâches dont l'ordonnancement conditionne la maîtrise par les éleveurs de la conduite de 
leur activité (Dedieu et al., 1998). Sur ce plan, nous nous sommes attachés à deux objectifs : qualifier l'organisation du travail dans les exploitations et étudier sa robustesse vis-à-vis d'aléas (en référence à la notion de « flexibilité opérationnelle ») ; explorer la « flexibilité stratégique » de l'organisation du travail, en privilégiant l'examen des changements éventuels intervenus dans l'orientation de la production bovine, dans la structure foncière de l'exploitation et dans la composition du collectif des permanents de l'exploitation.

- Le cinquième nous a permis, enfin, de collecter les informations nécessaires pour une analyse sociologique de l'action professionnelle des éleveurs visant à appréhender les processus délibératifs (Conein et Jacopin, 1994) en jeu dans la transformation des normes d'exercice de leur métier. Ces processus ont été appréhendés à deux niveaux. Nous nous sommes intéressés à la manière dont le système de relations des éleveurs et, notamment, la composition de leurs réseaux de dialogue professionnel (Darré, 1996) pouvait conditionner leur rapport au changement, rapport que nous avons donc cherché à expliciter. Nous nous sommes également attachés ${ }^{10}$ à voir comment le genre d'expérience professionnelle qui était le leur pouvait jouer sur ce rapport.

\section{Les éleveurs face à l'incertitude : des logiques diverses}

Le traitement de l'ensemble d'informations ainsi obtenues a d'abord consisté à qualifier le rapport au changement des éleveurs en nous appuyant sur les données de nos enquêtes sociologiques. Nous avons ensuite entrepris de mettre en relation les différents rapports au changement ainsi identifiés avec le fonctionnement effectif des exploitations.

10. En référence à la notion de transaction biographique proposée par Dubar (1991) dans son analyse de la construction des identités professionnelles.

\section{Deux « styles " de rapport au changement}

L'analyse du rapport au changement des éleveurs a été effectuée en combinant trois registres de lecture de leur discours. Nous avons d'abord relevé l'ensemble des énoncés dans lesquels ils formulaient un jugement sur les évolutions auxquelles ils étaient confrontés ou dans lesquels ils s'expliquaient sur la manière dont ils essayaient de répondre à ces évolutions ${ }^{11}$. Nous avons ensuite caractérisé les cadres spatio-temporels associés aux situations de changement décrites par les éleveurs, en examinant les moments (choisis/subis) qui leur correspondaient et l'étendue des problèmes que ces situations impliquaient selon eux de prendre en compte. Nous nous sommes enfin intéressés aux procédures mises en œuvre par les éleveurs dans ces situations. Cette démarche nous a permis de mettre en évidence deux styles de rapport au changement assez contrastés, « agir sur » et « faire avec ».

\section{" Agir sur "}

Pour les huit éleveurs relevant de ce premier cas de figure (groupe 1, éleveurs $\mathrm{n}^{\circ} 1,2,4$, $7,8,10,12,15)$, changer est associé à l'idée d'examiner un problème sous toutes ses faces avant de prendre une décision à laquelle il convient ensuite de se tenir. Il ne faut surtout pas changer d'optique au gré des aléas et, a fortiori, en temps de crise : il est important de se donner des objectifs clairs, de se fixer une ligne de conduite et de la respecter, envers et contre tout. Ces éleveurs se réfèrent à certaines normes explicitement formulées et revendiquées quant à ce que doit être l'élevage (un élevage " moderne», « professionnel »). Le passage à l'engraissement constitue, pour la plupart d'entre eux, le principal changement qu'ils ont opéré, à un moment présenté comme clé dans leur histoire (installation, modification de la composition du collectif de travail,

11. Avec une attention particulière à la signification qu'ils donnaient à la notion « d'adaptation ». 
reconstitution du cheptel après des problèmes sanitaires...). Leur discours valorise cette option du fait de la régularité et de la maîtrise de la production qu'elle permet. L'avenir de la profession, thème central dans la justification de leur comportement, tient selon eux à sa capacité à s'affirmer collectivement face aux autres maillons de la filière.

\section{" Faire avec »}

Pour les six éleveurs relevant de ce deuxième cas de figure (groupe 2, éleveurs $\mathrm{n}^{\circ} 3,5,6,11,13,14$ ), le changement - notion qu'ils relativisent fortement - est associé à l'idée de « faire de petits essais, pour voir » et à une évolution en continu pour s'adapter à la conjoncture. L'essentiel est de faire en sorte de ne pas se retrouver « coincé » dans des situations irréversibles. Il s'agit donc d'essayer des choses qui pourraient marcher, tout en se donnant la possibilité de revenir en arrière. Les exemples de changements que donnent ces éleveurs sont beaucoup plus variés que dans le premier cas. Ils portent sur la construction de bâtiments, le développement du recours à l'insémination artificielle, l'arrêt ou la reprise de certaines cultures et sont souvent associés à ce qui est présenté comme des contraintes : mise aux normes des bâtiments, climat, nature des sols... L'avenir de leur exploitation davantage que celui de la profession est ce qui constitue la toile de fond de leur discours.

\section{Quatre logiques types}

Nous avons affiné notre analyse en mettant en perspective ces deux styles de rapport au changement avec le fonctionnement des exploitations. Ce fonctionnement a été caractérisé en reconstituant la trajectoire des exploitations ${ }^{12}$ et en recoupant les différents points de vue disciplinaires mobi-

12. Trajectoire depuis l'installation de l'enquêté, appréhendée en termes d'évolution de la structure de l'exploitation et d'orientation et de réorientation éventuelle de production. lisés dans notre recherche pour rendre compte des pratiques des éleveurs. Les associations que nous avons pu établir entre un certain style de rapport au changement, une certaine trajectoire et un certain profil de pratiques ${ }^{13}$, nous ont alors permis de constituer quatre groupes dans notre échantillon, chacun correspondant à une logique particulière mise en œuvre par les éleveurs pour faire face aux aléas tout en défendant une certaine conception de leur métier se traduisant dans certains schèmes d'action privilégiés.

\section{Jouer sur la force d'un collectif professionnel organisé pour la production d'animaux " finis »}

Le premier groupe (groupe 1a, éleveurs $\left.\mathrm{n}^{\circ} 1,7,8,10,12\right)$ rassemble des éleveurs qui se sont installés depuis 5 à 20 ans sur des exploitations qui ont connu une augmentation de surface limitée relativement à celles de nos trois autres groupes. Ces exploitations se caractérisent également par une stabilité du collectif de travail (reposant essentiellement sur le couple) et une intensification de la production bovine, liée à la mise en place de l'activité d'engraissement pour tout ou partie des $\operatorname{animaux}^{14}$. Globalement, la stratégie des éleveurs concernés vise à réduire la sensibilité de leur exploitation aux aléas en s'appuyant sur des contrôles fréquents, avec des prises d'informations nombreuses et une gestion très individualisée des animaux (par opposition à une conduite groupée, en lots). Ces éleveurs cherchent à maîtriser les processus biologiques, par

13. Profil correspondant à un certain mode de conduite technico-économique de l'élevage, à une certaine situation de travail et à certaines caractéristiques sociologiques des éleveurs (une certaine composition de leur système de relations professionnelles et sociales et un certain type de rapport aux Organisations professionnelles agricoles (OPA), notamment).

14. Le choix de l'intensification tient souvent à une absence de possibilité d'agrandissement de 1'exploitation. Il tient aussi, on va le voir, à certaines options idéologiques... 
la sélection (génétique), l'allotement et l'alimentation. Cette maîtrise technique leur permet de produire des animaux répondant aux attentes de la filière (âge, conformation, période de l'année). Les ventes sont étalées sur plus de six mois et la proportion d'animaux vendus sous signes officiels de qualité est relativement importante. Fortement impliqués dans l'appareil des OPA et disposant d'un réseau de relations professionnelles à la fois dense et étendu, les éleveurs de ce groupe mettent l'accent sur la vente d'animaux « finis » qui représentent toujours plus d'un tiers des effectifs commercialisés. Très marqués par la vision de ce que doit être un élevage charolais « moderne » portée par les organisations collectives auxquelles ils appartiennent - organisations affichant fortement la nécessité de rompre avec une logique de « cueillette » pour aller vers une véritable activité de production impliquant le passage à l'engraissement pour conserver le maximum de valeur ajoutée -, ils s'investissent dans la recherche d'une meilleure valorisation des animaux produits (projets de vente directe, labels, projet d'Appellation d'Origine Contrôlée). Ils se montrent très critiques à l'égard de ceux de leurs collègues qu'ils considèrent vendre n'importe quoi, n'importe quand et à n'importe qui, sans engraisser les animaux, en déstabilisant le marché par une production et des ventes en « dents de scie ». En contrepartie de l'intensification de la conduite technique, les marges de manoeuvre en termes de structure sont très limitées, voire nulles. Les petites dimensions de ces structures (à une exception près) rendent, cependant, la situation confortable en termes de charge de travail. Si une partie des tâches est effectuée grâce à du bénévolat ou à de l'entraide, il n'est jamais fait référence à un recours éventuel à de la main-d'œuvre salariée. La marge de manœuvre représentée par le « temps disponible calculé » annuel (temps restant une fois les tâches liées à l'élevage et aux surfaces fourragères correspondantes réalisées) ${ }^{15}$ est correcte dans tous les cas.

\section{Miser sur la taille de l'exploitation}

Un deuxième groupe (groupe $1 \mathrm{~b}$, éleveurs $\left.\mathrm{n}^{\circ} 2,4,15\right)$ rassemble des éleveurs dont le jugement, assez ambivalent, sur le lien qu'il y aurait entre maîtrise de la production et engraissement est beaucoup plus nuancé que celui des précédents. L'engraissement ne constitue pas selon eux la solution unique aux problèmes rencontrés par l'élevage allaitant charolais. S'ils se réfèrent bien à cette option - le fait qu'ils se définissent, pour certains, comme de « mauvais engraisseurs » semblant indiquer une certaine subordination à l'égard de la position, dominante dans le champ professionnel, des éleveurs du premier groupe -, ils revendiquent la pertinence « aussi »d'une logique d'agrandissement et ils considèrent que cette logique est, à long terme, celle qui permettra aux éleveurs de s'en sortir. Ce qui les caractérise, c'est la poursuite d'un objectif qui peut se résumer par «faire du nombre », indépendamment de l'évolution de la conjoncture. Installés depuis 5 à 15 ans, ils apparaissent très soucieux de «se faire une place » dans un monde charolais où la logique du rang à tenir et un certain éthos de puissance restent très prégnants. Ils ont cherché et réussi à s'agrandir fortement, leurs trajectoires étant marquées par des changements assez radicaux dans l'appareil de production initial (cheptel, bâtiments, surfaces) dont ils ont hérité. Cet agrandissement s'accompagne d'un investissement en matière de génétique, avec un souci de progresser dans ce domaine. Les éleveurs ici concernés considèrent l'accès à une " grosse structure » comme une sorte de préalable. Les problèmes induits par la conduite d'un tel type d'exploitation - amélioration de la conformation des animaux après une phase

15. Cet indicateur a été calculé à partir de la méthode $\mathrm{du} \ll$ Bilan Travail » (Dedieu et al., 2000). 
de croissance en interne des effectifs (peu de sélection possible) ou bien adaptation du système fourrager aux besoins accrus du troupeau en ressources alimentaires - ne sont envisagés et traités que lorsque le but ainsi fixé a été atteint. Dans ce groupe, la priorité étant la taille du troupeau de vaches (nombre de vêlages annuels), la proportion d'animaux finis est toujours inférieure à un tiers. Un tel choix se traduit par de fortes contraintes d'organisation du travail, notamment en hiver (problèmes de places en bâtiments, surveillance des vêlages, soins aux veaux). Ces contraintes sont d'autant plus marquées que le collectif de travail se caractérise, dans ces exploitations, par une certaine instabilité (départ des parents, entrée ou sortie de l'épouse). Pour y faire face, deux éleveurs sur trois ont recours, outre à l'entraide dans le cadre de réseaux de proximité, au salariat, ce qui leur permet de tenir avec une marge de manœuvre suffisante. Ne se sentant pas vraiment soutenus par «la profession» et n'étant pas toujours «bien vus », localement, par les autres éleveurs (du fait de leur choix de jouer la carte de l'agrandissement, mais aussi du fait d'un parcours souvent chaotique - impossibilité de prétendre aux aides à l'installation du fait d'un diplôme d'étude insuffisant, reconstitution d'un cheptel suite à la brucellose... -) ces éleveurs s'impliquent néanmoins dans les OPA, mais moins par conviction, semblet-il, que par intérêt bien compris (pour se tenir au courant).

\section{Maintenir un système " robuste » et autonome}

Le troisième groupe (groupe 2a, éleveurs $\left.\mathrm{n}^{\circ} 5,6,13\right)$ rassemble des exploitants pour lesquels la référence à la « tradition charolaise » est très centrale. Leurs trajectoires correspondent toutes à des transmissions de père en fils, avec des modes de fonctionnement se maintenant au fil des générations. Il s'agit de systèmes extensifs reposant sur un agrandissement des surfaces mené en parallèle à une augmentation du troupeau ou sur une valorisation de la production herbagère par certaines catégories d'animaux privilégiées (bœufs), en limitant délibérément l'effectif total du troupeau. Toutes ces exploitations sont très spécialisées, sans production complémentaire hors élevage bovin. La logique de production des éleveurs de ce groupe repose sur l'association d'un faible taux de chargement avec une conduite des animaux fondée avant tout sur l'expérience, ce qui a fait ses preuves par le passé et qu'il faut donc transmettre. Cette conduite vise essentiellement à laisser s'exprimer les régulations « naturelles » permises par la synchronisation des cycles biologiques des animaux et des surfaces : organisation par exemple de la saison de reproduction au pâturage coïncidant avec la pousse de l'herbe au printemps (vêlages de fin d'hiver). Ces éleveurs considèrent que l'avenir de leur exploitation dépend, à la fois, de leur capacité à maîtriser l'organisation de leur système d'élevage et de leur capacité à garder la main sur la commercialisation des animaux. Leur objectif principal est de préserver leur autonomie : autonomie dans l'alimentation du troupeau, autonomie vis-à-vis des structures d'aval - et, plus largement, de tout l'appareil d'encadrement professionnel agricole -, mais aussi autonomie dans le travail. Les exploitations concernées se caractérisent, en effet, par la faiblesse des contributions extérieures au noyau familial, l'apprentissage in situ étant fortement valorisé et les réseaux de relations professionnels de ces éleveurs apparaissant assez peu étendus, alors qu'elles correspondent à des systèmes pourtant exigeants en suivis. En conséquence, lorsqu'il s'agit d'exploitations de taille importante (relativement à la maind'œuvre présente), la marge de manœuvre en temps disponible est assez faible. Dans tous les cas, cependant, l'autonomie - technique, en particulier - dont disposent les éleveurs de ce groupe du fait de la souplesse structurelle de leurs exploitations (faible chargement), leur permet de faire des ajustements à court terme sans changer fondamentale- 
ment le fonctionnement du système et de maintenir le cap sur le moyen et le long terme.

\section{Saisir les opportunités}

Un quatrième groupe, enfin, réunit des éleveurs (groupe $2 b$, éleveurs $\mathrm{n}^{\circ} 3,11,14$ ) qui, à la différence de tous les autres enquêtés, ne manifestent pas d'attachement très marqué au monde de l'élevage et revendiquent même une certaine distance à l'égard de l'agriculture, activité qu'ils exercent tout en cherchant à ne pas y être «pris » et dont certains n'excluent pas la possibilité de l'abandon. Tous ont eu des expériences professionnelles hors agriculture avant de s'installer et les exploitations concernées se caractérisent par une tendance marquée à la diversification, tendance qui s'est accrue au fil du temps. L'un d'entre eux (habitant dans un bourg) se définit avant tout comme un « cultivateur ». Un autre associe à sa production de bovins un élevage de poulets et des cultures de vente. Le troisième, qui a travaillé dans l'informatique avant de reprendre (pour des raisons complexes, mêlant attachement et rejet de la tradition dans une forme de «néo-ruralisme » assez ambivalente) une exploitation familiale dans laquelle l'élevage équin était aussi important que l'élevage bovin, apparaît plus attiré par la viticulture (qu'il exerce aussi) que par cette activité. Le raisonnement de ces éleveurs, ou plutôt de ces « entrepreneurs », est guidé par le souci d'opérer des choix qui n'engagent pas le système bovin en profondeur, mais permettent de répondre aux aléas de la demande, c'est-à-dire de saisir les opportunités de vente quand elles se présentent (variation des cours selon les catégories d'animaux, par exemple). En matière d'élevage, ils n'ont pas de projet de production prédéfini, mais ils sont plutôt dans une logique d'adaptation permanente aux fluctuations de cette demande. Cela ne signifie pas qu'ils ne maîtrisent pas la conduite de leur activité, mais, au contraire, qu'ils sont capables d'ajustements et de réorientations assez rapides sur les catégories produites (finition ou non). Cela se traduit par une gamme diversifiée de produits commercialisés. Les structures relativement favorables qui sont les leurs (surface, chargement) leur offrent également des marges de manœuvre importantes avec, en contrepartie, une situation tendue en termes de charge de travail. Deux sur trois y font face avec du salariat. Assez peu insérés dans le monde des OPA (ou, plus exactement, limitant leurs adhésions à des organismes à vocation d'abord économique), leurs réseaux professionnels sont essentiellement composés de «personnes ressources » appartenant à des univers sociaux relativement variés avec lesquelles ils entretiennent des relations privilégiées en fonction de l'intérêt qu'elles présentent au regard de leur souci de «diversifier leur mise ».

\section{Enseignements de l'étude et conséquences en matière de développement}

La manière dont les éleveurs répondent aux événements qui affectent leur activité apparaît donc loin d'être homogène ${ }^{16}$. La crise de l'ESB auquel l'élevage bovin a été confronté en 2001 a ainsi suscité des réactions différentes chez les exploitants enquêtés. Seuls ceux du premier des quatre groupes que nous avons identifiés (groupe 1a) ont été vraiment affectés. Ayant investi dans la production d'animaux finis, ils ont été directement touchés par la chute de la consommation qui a résulté de cette crise, leurs systèmes d'élevage, très poussés tech-

16. Cette variété est d'autant plus à souligner que le petit nombre d'exploitations enquêtées et les biais introduits par la procédure adoptée pour constituer cet échantillon même (des exploitations choisies sur une liste fournie par des responsables et/ou des techniciens de structures de commercialisation) ont sans doute contribué à réduire le spectre des différents cas de figure effectivement pris en compte dans notre étude. 
niquement, ne leur offrant que peu de marges de manœuvre. S'ils ont envisagé la possibilité d'une certaine diversification de leur activité - soit en se lançant dans de la vente directe de viande bovine, soit en mettant en place ou en développant des productions complémentaires -, leur réaction s'est surtout traduite par un appel à une meilleure organisation des producteurs. Pour eux, en effet, seule une mobilisation collective était à même de permettre d'obtenir le poids politique nécessaire pour peser sur le marché et restaurer la confiance des consommateurs. Dans les trois autres groupes, par contre, la crise de l'ESB n'a pas suscité de réactions particulières. Pour le second (groupe $1 \mathrm{~b}$ ), ce phénomène résulte du fait que les conséquences de cette crise sont restées relativement mineures au regard de difficultés structurelles tenant à la trajectoire d'évolution dans laquelle les exploitants correspondants étaient engagés (agrandissement, augmentation du troupeau). Mais leur absence de réaction marquée renvoie aussi à la logique même qui est la leur, faire montre d'une certaine capacité à « encaisser » (quel qu'en soit le degré de réalité, « ça passe ou ça casse »...) étant un élément central de leur modèle d'excellence professionnelle. Pour les éleveurs du troisième groupe (2a), cette absence de réactions est surtout due au fait que la crise ne les a effectivement pas atteints (animaux vendus avant les effets de la crise ou catégories produites non concernées). Mais elle se comprend bien, là encore, au regard de leur souci d'autonomie : leur système de production est précisément «formaté » pour absorber les aléas, quels qu'ils soient. Quant au quatrième groupe (2b), si les éleveurs qui en relèvent se trouvaient confrontés - comme ceux du deuxième - à des problèmes tels que la crise ne les a pas spécifiquement perturbés ${ }^{17}$, la conception

17. Problèmes, dans leur cas, autant conjoncturels que structurels. qu'ils ont de leur activité explique également cette attitude. Se définissant comme des entrepreneurs, le risque fait partie pour eux de cette activité et l'important est de savoir en jouer au mieux davantage que de s'en préserver à tout prix au nom d'un attachement, qu'ils dénoncent, à un monde de l'élevage bovin allaitant dont les contours et les règles de fonctionnement seraient immuables.

Cette diversité de réactions montre que les éleveurs répondent aux évolutions de leur secteur suivant la façon dont celles-ci affectent objectivement leur système d'exploitation, mais aussi en fonction de certaines normes relatives à ce qu'est et de ce que doit être leur métier. La perception qu'ils ont ainsi de leur environnement et de ce qui change dans cet environnement n'est pas identique. Pour certains (groupe 1a), particulièrement sensibles, de par leurs responsabilités professionnelles et leurs réseaux de relations étendus et diversifiés, à la «place de l'agriculture dans la société » et fortement investis dans la promotion d'une conception de l'élevage mettant l'accent sur la maîtrise technique des opérations de production et le contrôle de la filière, les évènements auxquels ils étaient confrontés au moment de nos enquêtes étaient interprétés comme une crise majeure, mettant en évidence des problèmes de coordination et de communication devant être impérativement traités. Pour d'autres (groupes 1 b et $2 a$ ), se caractérisant par univers social de référence plus homogène - assez exclusivement centré sur le monde de l'élevage - et attachés à la défense de la «tradition charolaise » (tradition qu'ils n'envisagent pas dans les mêmes termes, cependant), ces évènements étaient vus plutôt comme des aléas parmi d'autres, aléas dont il convient, autant que faire se peut, de se tenir à distance. Et pour d'autres encore (groupe 2b), occupant une position un peu en marge du monde de l'élevage, ils étaient considérés comme des signaux « naturellement » à prendre en 
compte dans un processus d'ajustement continu d'une activité de production économique relativement dissociée de leurs engagements personnels et civiques.

Mais ce qui est également à souligner, c'est que ces diverses réponses ne sauraient être étalonnées sur une échelle unique «d'adaptation». Si les exploitations que nous avons enquêtées se trouvaient dans des situations plus ou moins favorables, ces situations ne s'ordonnent pas suivant un gradient permettant de considérer les différentes logiques que nous avons mises en évidence comme plus ou moins appropriées. Tout se passe plutôt comme si les éleveurs disposaient de différents ressorts d'action - l'organisation collective, la taille de l'entreprise, la robustesse du système de production, la diversification des produits - hiérarchisés et mobilisés en fonction de leurs ressources techniques, économiques mais aussi sociales, l'activation et la combinaison de ces ressorts générant, dans chaque cas, des atouts et des difficultés spécifiques.

Cette complexité tient au fait que les évolutions du contexte d'exercice de l'élevage ont « réellement» différentes significations possibles entre lesquelles il n'est pas évident de trancher et qui doivent être éprouvées. Le devenir de ce secteur ne peut donc pas être considéré comme joué d'avance. Il dépend, au moins pour partie, des anticipations et des actes posés par des éleveurs qui visent un certain état de l'élevage et, surtout, de l'issue de la confrontation des différents « projets » dont sont porteurs ces éleveurs, c'est-à-dire, aussi, du rapport de forces susceptible de s'établir entre les tenants de ces différents projets. De ce point de vue, ce qu'il convient de faire est bien « à trouver », et c'est en cela - comme indiqué dans l'exposé de notre problématique - qu'une analyse des transformations de l'élevage en termes de processus d'invention nous semble importante à prendre en compte. En situation d'incertitude, la pertinence et l'efficacité des réponses que les éleveurs élaborent pour faire face à un nouveau contexte de production ne peuvent, en effet, s'apprécier au regard de la plus ou moins grande conformité de ces réponses à un modèle posé $a$ priori. Ce qui est déterminant c'est la capacité des acteurs à réfléchir les compromis particuliers qu'ils ont établis ou qu'ils cherchent à établir entre les différents ressorts sur lesquels ils peuvent jouer pour faire face à ce qui leur arrive.

Si l'on s'interroge alors sur ce que devrait être un accompagnement des éleveurs à même de faciliter leur " adaptation » aux évolutions de leur environnement, les résultats de notre étude amènent à envisager les choses suivant une perspective un peu différente de celle généralement adoptée par les organismes de développement intervenant dans ce secteur. En rupture avec l'accent assez exclusif que ceux-ci mettent sur la première des quatre logiques que nous avons dégagées, ce sont les moyens, pour les éleveurs, de se situer au regard de la diversité des options qui s'offrent à eux qu'il importerait de renforcer. Nos résultats pourraient ainsi fournir des bases pour l'élaboration d'une grille d'évaluation multidimensionnelle de leurs pratiques, un tel outil - restant à élaborer et dont la figure 1 qui suit ne se veut que donner une idée - étant destiné à leur permettre de se positionner et de se projeter dans l'avenir en examinant les conséquences de leur engagement dans telle ou telle voie au regard de l'ensemble des ressorts susceptibles de leur permettre d'atteindre un certain équilibre entre réactivité et stabilité de leur système d'exploitation. 


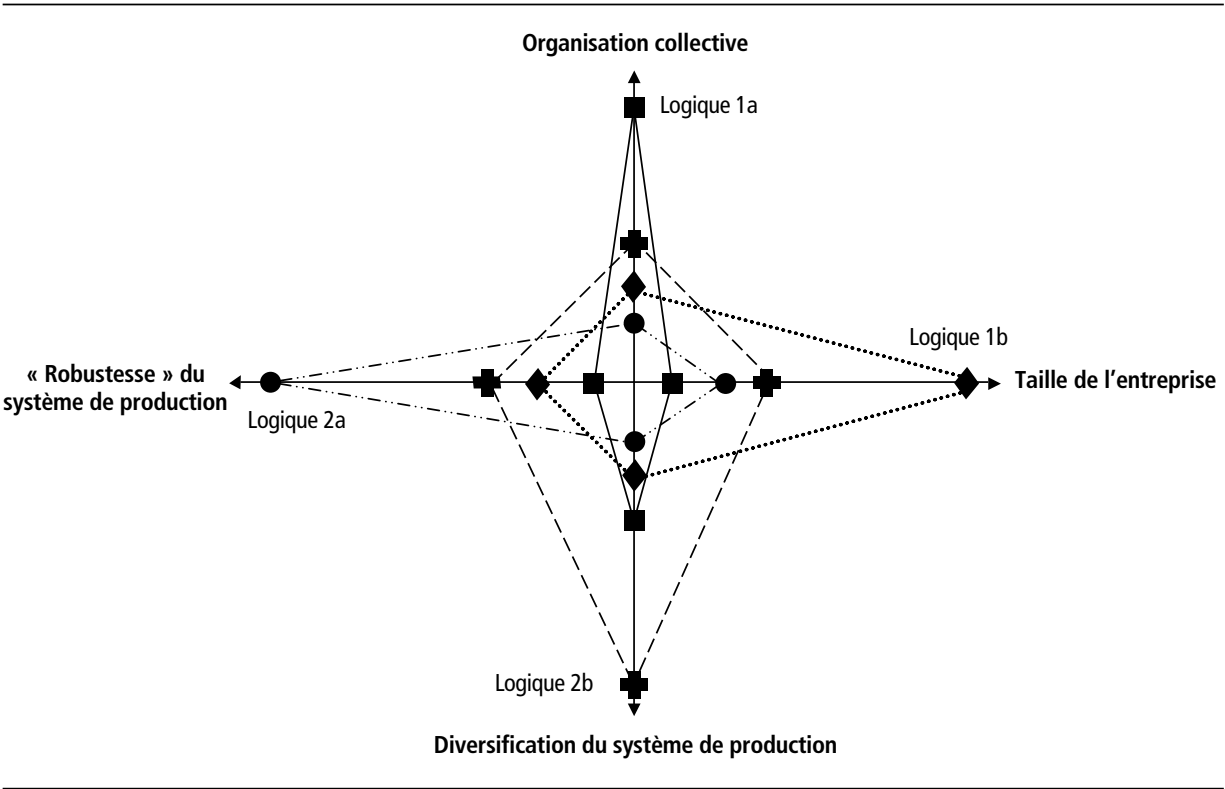

Raisonner en termes d'invention autant que d'adaptation conduit également à proposer une autre inflexion dans la façon d'aborder la question de l'accompagnement des éleveurs. Ce qu'indique aussi notre étude, c'est que les compétences - les savoirs et les savoir-faire techniques, économiques, organisationnels et relationnels sur lesquelles repose une telle invention sont distribuées entre différents types d'éleveurs. Si la crise que traverse actuellement ce secteur peut être considérée comme une crise « globale », ce qu'elle remet exactement en cause ne peut être saisi, en effet, qu'au travers des conséquences différenciées qu'elle induit dans le monde de l'élevage. C'est, en quelque sorte, la somme des expériences de ce qu'elle implique pour chacune des composantes ou, pour nous référer ici à la sociologie des professions, de chacun des « segments » (Hughes, 1996) constitutifs de ce monde, qui peut permettre de prendre la mesure de ce qu'elle recouvre précisément. Dans ces conditions, la validité des réponses qu'appelle cette crise ne peut être appréciée que pour autant que l'en- semble des solutions envisageables donne lieu à une exploration et à une mise à l'épreuve effectives.

Chercher à augmenter la capacité d'action des éleveurs suppose alors davantage qu'une amélioration de leur réflexivité individuelle. Ce qui est tout autant à développer, c'est le débat - et la productivité du débat - sur ce que doit être aujourd'hui leur métier. $\mathrm{Ce}$ débat concerne des éleveurs aux positions très différentes dans leur champ professionnel et dotées d'une légitimité et d'une reconnaissance assez inégales. Un investissement particulier est ainsi nécessaire pour qu'il puisse être instauré et mené à son terme. Comme on l'a vu, ainsi, les logiques d'action des éleveurs de nos groupes $2 \mathrm{a}$ et $2 \mathrm{~b}$ ne manquent pas d'une certaine pertinence, alors même que les premiers sont généralement considérés comme attachés à un modèle d'excellence professionnel « dépassé » et que les seconds sont jugés quelque peu «marginaux ». Trouver les moyens d'une réévaluation effective des positions qu'ils défendent est donc aussi un impératif de développement. 


\section{RÉFÉRENCES BIBLIOGRAPHIQUES}

Barbier J.-C., Nadel H. La flexibilité du travail et de l'emploi; Flammarion, Paris, 2000, 120 p.

Boltanski L., Chiappello E. Le nouvel esprit du capitalisme. Gallimard, Paris, 1999, $843 \mathrm{p}$.

Cohendet P., Llerena P. Flexibilité et modes d'organisation. Revue française de gestion, Lavoisier, Paris, 1999, p. 72-79.

Conein B., Jacopin E. Action située et cognition, le savoir en place. Sociologie du Travail, Elzevier, Paris, 1994, n 36/4, p. 475-500.

Cristofini B., Deffontaines J.-P., Raichon C., De Verneuil B. Pratiques d'élevage en Castagniccia. Exploration d'un milieu naturel et social en Corse ; Études Rurales, EHESS, Paris, 1998, n 71-72, p. 89-109.

Darré J.-P. L'invention des pratiques dans l'agriculture. Vulgarisation et production locale de connaissance. Karthala, Paris, 1996, 194 p.

Dedieu B., Chabosseau J.-M., Willaert J., Benoit M., Laignel G. L'organisation $d u$ travail dans les exploitations d'élevage : une méthode de caractérisation en élevage ovin du Centre-Ouest. Études et Recherches sur les Systèmes Agraires et le Développement, INRA, Paris, 1998, n 31, p. 63-80.

Dedieu B., Chauvat S., Servière G., Tchakérian E. Bilan travail pour l'étude du fonctionnement des exploitations d'élevage: Méthode. Institut de l'Élevage / INRA, Collection Lignes, Paris, 2000, 27 p.

Dégrange B. La mise à l'épreuve d'une profession. Le travail de redéfinition du métier d'éleveur charolais. Thèse de doctorat de Sociologie, Université Lumière / Lyon 2, 2001, 396 pages.
De Gaulejac V. La société malade de la gestion. Idéologie gestionnaire, pouvoir managérial et harcèlement social. Seuil, Paris, 2005, 276 p.

Dubar C. La socialisation. Construction des identités sociales et professionnelles. Armand Colin, Paris, 1991, 276 p.

Hughes E.-C. Le regard sociologique. Essais choisis. EHESS, Paris, 1996, 344 p.

Landais E., Deffontaines J.-P. Les pratiques des agriculteurs. Point de vue sur un courant nouveau de la recherche agronomique. Études Rurales EHESS, Paris, $1988, n^{\circ} 109$, p. 125-158.

Lémery B. Les agriculteurs dans la fabrique d'une nouvelle agriculture. Sociologie du Travail, Elzevier, Paris, 2003, $\mathrm{n}^{\circ}$ 45/1, p. 9-25.

Lémery B., Bardey H., Brossier J., Dedieu B., Dégrange B., Ingrand S. Evolutions de la filière bovine et «flexibilité » des exploitations d'élevage. Etudes de cas dans le bassin allaitant bourguignon. Rapport final au Comité Scientifique du Programme PSDR Bourgogne, INRA-LISTO, Dijon, 2004, $61 \mathrm{p}$.

Périlleux T. Les tensions de la flexibilité. Desclée de Brouwer, Paris, 2001, 220 p. Sébillotte M., Soler L.-G. Les processus de décision des agriculteurs, dans Brossier et al. éd, Modélisation systémique et système agraire. Décision et organisation. INRA, Versailles, 1990, p. 93-101.

Sennett R. Le travail sans qualités. AlbinMichel, Collection 10/18, Paris, 2000, $211 \mathrm{p}$.

Tarondeau J.-C. Approches et formes de flexibilité. Revue française de gestion, Lavoisier, Paris, 1999, p. 66-71. 